\title{
Maintenance of certification: part 2-continuous certification
}

\author{
Joshua A Hirsch, ${ }^{1}$ Gary J Becker, ${ }^{2}$ Colin P Derdeyn, ${ }^{3}$ Mahesh V Jayaraman, ${ }^{4}$ \\ Vincent C Traynelis, $^{5}$ Philip M Meyers ${ }^{6}$
}

${ }^{1}$ NeuroEndovascular Division Massachusetts General Hospital, Harvard Medical School, Boston, Massachusetts, USA

${ }^{2}$ American Board of Radiology, Tuscon, Arizona, USA ${ }^{3}$ Mallinckrodt Institute of Radiology and the Departments of Neurology and Neurosurgery, Washington University School of Medicine, St Louis, Missouri, USA ${ }^{4}$ Rhode Island Hospital, Warren Alpert School of Medicine at Brown University, Providence, Rhode Island, USA

${ }^{5}$ Rush University Medical Center, University

Neurosurgery, Chicago, Illinois, USA

${ }^{6}$ Department of Neurological Surgery, Neurological Institute, New York, New York, USA

\section{Correspondence to} Dr J A Hirsch,

NeuroEndovascular Division, Massachusetts General Hospital, Harvard Medical School, Boston, MA 02114, USA:

Hirsch@snisonline.org

Received 5 February 2013 Accepted 18 February 2013 Published Online First 15 March 2013

To cite: Hirsch JA, Becker GJ, Derdeyn CP, et al. J Neurolntervent Surg 2014:6:156-160.

\section{INTRODUCTION}

In the first article of this series, we explored the historical development of medical specialty societies, and how this process ultimately resulted in the formation of the American Board of Medical Specialties (ABMS). The Maintenance of Certification (MOC) program grew out of this association as a means to ensure continuous improvement throughout each physician's career in a specialty chosen area of specialization.

Neurointervention is somewhat unique in that it enjoys the participation of physicians from three different specialty boards: radiology, neurosurgery, and neurology. In order to understand the MOC process, the reader needs some information about the primary certification process of the individual boards. Here, we will review the primary certification and the various MOC requirements that neurointerventionalists will be exposed to through their specific parent organization: American Board of Radiology (ABR), American Board of Neurological Surgery (ABNS), and American Board of Psychiatry and Neurology (ABPN). We relied heavily on the individual specialty board websites, which will be cited throughout this text, as well as direct communication with the representatives of the three boards. In the event that the question should arise, the authors note that the order of discussion was chosen in a random fashion.

\section{AMERICAN BOARD OF NEUROLOGICAL SURGERY \\ Primary certification}

The ABNS was approved as an examining board in 1940 by the action of the Advisory Board for Medical Specialties, a precursor body to the ABNS. Primary certification by the ABNS occurs following completion of an approved educational training program, and involves both a written and an oral examination. ${ }^{1}$ Below are detailed the residency criteria, effective from 7 January 2013.

Neurosurgical residency is 7 years in duration. Training begins when a full time resident enrolls in a program recognized by the ABNS and accredited by the Accreditation Council for Graduate Medical Education (ACGME) via its Residency Review Committee (RRC) for Neurological Surgery. During the first 18 months of education, residents must have at least 3 months of basic clinical neuroscience education and at least 3 months of critical care education applicable to the neurosurgical patient. There also must be 6 months of structured education in general patient care, which usually includes rotations on trauma, vascular, and other related surgical services. At least 3 months must be devoted to clinical neurology as a full time assigned resident in an RRC approved neurology program.

Fifty-four months of clinical neurological surgery education must be completed at the primary clinical site or at an approved participating site. A minimum of 21 months of neurological surgery education must occur at the primary clinical site. The resident is given progressive responsibilities, culminating in 12 months at the chief resident level. It should also be noted that the RRC sets minimum case requirements for key procedures that each resident is expected to reach or succeed by the conclusion of the chief year. These requirements include arteriography and endovascular therapy for tumor and vascular lesions.

The remaining months must be used for elective clinical education and/or research. Future endovascular neurosurgeons often use that time to perform part/all of their endovascular training. Neurosurgical residents interested in formal endovascular training should note that a premium is placed on obtaining endovascular training within the same program to which they were originally matched. Training of any type at an institution outside of the parent program and its affiliated hospitals will not count toward meeting primary certification requirements unless credit is requested from the RRC in advance by the program director and approved in writing. The primary examination is a key step in achieving certification by the ABNS. It is designed to both evaluate knowledge and provide a basis for continued learning. The examination is administered in mid-March. Most program directors require passage before the chief resident year.

Prior to accepting a candidate into the certification process, the board requires a statement from the individual's program director to the effect that he or she has satisfactorily performed the minimum training requirements and is prepared to enter into the independent practice of neurosurgery, successful completion of the written primary examination, and submission of 150 consecutive cases with follow-up for review. Permission for oral examination will not be granted to a neurosurgical resident until the candidate has met these and other requirements established by the ABNS. A candidate who passes the oral examination becomes a certified diplomate of the ABNS.

A candidate who fails his or her first oral examination may sit for re-examination a second or, if necessary, a third time. Both the second and third attempts must be within 7 years of completing residency. A candidate who has failed the oral examinations on three occasions is no longer considered 
eligible for certification. He or she must retake and pass the primary examination before re-entering the certification process. $^{2}$

\section{AMERICAN BOARD OF RADIOLOGY Primary certification}

The ABR was conceived in the early 1930s and incorporated in 1934. The primary charge of the board was to provide examinations and certify physicians who sought recognition as radiologists. The ABR found it necessary to define residency training requirements which led it to co-sponsor RRCs for diagnostic radiology and radiation oncology. As practices grew and evolved, radiation oncology and diagnostic radiology developed separate training programs, and both lengthened residency to 4 years. Medical physics has been and remains an important part of the training of radiologists until this day. ${ }^{3}$

Historically, the ABR issued lifetime certificates. In 1994, the ABR began to offer time limited certificates for areas of additional qualification. Most relevant to the Society of NeuroInterventional Surgery, a time limited (10 year) certificate of added qualification (CAQ) was offered in neuroradiology. Since 2002, only time limited certificates have been offered in diagnostic radiology as well. To obtain primary certification in diagnostic radiology, the trainee must first take and pass a written examination, one portion of which is clinical and the other portion is dedicated to radiologic physics. This is followed by a trip to Louisville, Kentucky, where qualified candidates undergo a half day oral, case based, examination. Typically, this occurs during residency, which diminishes productivity during the fourth year. Passing that oral examination is the final step in achieving board certification.

As of 2013, primary certification in diagnostic radiology will entail first successfully completing a computer based diagnostic radiology comprehensive core examination. The new computer based certifying examination, which will be administered 15 months after completion of training, will be given for the first time in October 2015.

The core examination, which replaces the current 'written' (computerized) examination, will be taken 36 months after the beginning of radiology residency training. It will be an image rich, computerized examination. The first administration of the core examination will be offered in September 30-October 4, 2013, for residents who began their training in 2010, and the test will ultimately be offered twice yearly. This 2 day examination will test knowledge and comprehension of anatomy, pathophysiology, and radiological physics. Eighteen categories will be covered in the core examination, with physics integrated into each category. The physics component will be separately scored and must be passed.

Although final determination of the grading method will depend on analysis of the June 2013 core pilot examination administration, initial proposals for scoring examinations have been drafted. As of today, if a candidate fails 1-5 categories in the 18 portion core examination, he/she will have 'conditioned' the examination. Conditioning necessitates a repeat examination in the up to five categories that were failed. If a candidate fails more than five categories, the entire examination must be repeated.

Candidates have 6 years from the end of their training to successfully complete the initial certification process (both core and certifying examinations) and describe themselves as 'board eligible'.

The certifying examination, which replaces the current oral examination, will be taken 15 months after completion of diagnostic radiology residency as opposed to during training. The core examination must be unconditionally passed before a candidate is eligible to take this certifying examination. The examination will emphasize synthesis of information, differential diagnosis, and patient management. Aspects of physics and basic sciences that are important in imaging will be included on the examination. The examination will take $5 \mathrm{~h}$ and will be offered twice yearly. It consists of a total of five modules.

The certifying examination will have two modules that must be taken by all examinees. The first is based on what the ABR is calling 'non-interpretive skills'. These include general topics of importance to the practice of radiology, such as radiation safety, recognition and management of contrast reactions, error prevention, communication skills, professionalism, ethics, and other aspects of practice. A non-interpretive skills syllabus is available on the ABR website. The second module is 'essentials of diagnostic radiology' which includes fundamental basic knowledge, such as recognizing emergency or critical medical conditions.

The certifying examination will also include three modules in clinical practice areas selected by the individual, based on training, experience, and practice emphasis (one, two, or three different practice areas may be selected). Neurointerventional radiologists, for example, might choose neuroradiology for all of these modules. The examination will be scored as pass or fail, and feedback will be provided to examinees. The two required modules must each be passed individually, and the elective modules must be passed as a group. If any of these three decisions is 'fail', the entire examination must be retaken. ${ }^{4}$

CAQs became available to neuroradiologists in 1994. These CAQs (now known as subspecialties) were always a 10 year, time limited certificate. Many neurointerventional radiologists chose to pursue such certification as an additional instrument beyond board certification in diagnostic radiology. ${ }^{5}$

\section{AMERICAN BOARD OF PSYCHIATRY AND NEUROLOGY Primary certification (vascular neurology)}

The ABPN requires that physicians have a valid medical license to practice medicine. This is true throughout the initial certification and MOC process. Physicians who are board certified by the ABPN will have successfully completed an approved training program and an evaluation process assessing their ability to provide quality patient care in their (sub)specialty. This evaluation process includes an initial certification examination on completion of the training program and evaluation.

The ABPN website indicates that ..."Patients can be assured that an ABPN board certified neurologist or child neurologist has specialized skills and knowledge to diagnose and treat specific problems and to provide medical management for a range of problems, including emergencies and long term care of chronic neurological disorders". These disorders include stroke among others. ${ }^{6}$

For purposes of this review, we will focus on certification in vascular neurology. The ABPN, working with the ABMS, established a Committee on Certification in the Subspecialty of Vascular Neurology in 2003. According to the ABPN website, "this was done to officially establish the field of vascular neurology as a definite area of subspecialization in neurology". It is further meant to provide a means of identifying properly trained and experienced vascular neurologists.

A Committee on Certification, a subsection of the ABPN, controls the mechanics of becoming certified in vascular neurology. Applicants for certification in vascular neurology must be board certified in the primary specialty of neurology by the end of the year prior to the examination. Following a grandfathering 
period (that ended in 2009), all applicants need documentation of having successfully completed 1 year of ACGME accredited fellowship training in vascular neurology. It is specified that this training did not begin during residency training. Stated differently, exposure to vascular neurology during one's basic neurology residency does not count toward the 1 year of training. All licensing and training requirements must be met by June 30 of the year of the examination.

Interestingly, the required 1 year of specialized training in vascular neurology does not need to be completed on a full time basis. It may be completed on a part time basis as long as efforts are not less than $50 \%$ of the applicant's time. Credit is not typically given for periods of training lasting less than 1 year, although certification is considered on an individual basis.

Applicants take a computerized, 200 question, multiple choice examination. According to the website, "candidates are assessed in basic science aspects of vascular neurology, prevention, risk factors and epidemiology, clinical features of cerebrovascular diseases, evaluation of the patient with cerebrovascular disease, causes of stroke, treatment of patients with stroke, and recovery, regenerative approaches, and rehabilitation".

There are fairly recent changes that have been implemented for applicants who are going to become newly certified. As of January 1, 2012, ABPN will require a physician to become board certified within 7 years following successful completion of ACGME accredited or ABPN approved residency training in their primary specialty or ACGME accredited subspecialty. Graduates can take the ABPN certification examination as many times as offered during the 7 year period. Thus individuals who have completed an accredited residency program prior to January 1, 2012, will have until January 1, 2019 to become board certified. ${ }^{7}$

\section{MAINTENANCE OF CERTIFICATION}

We will now explore MOC programs for the three parent organizations of neurointerventional physicians. There are four critical components to MOC:

1. Professional standing

2. Commitment to lifelong learning and periodic selfassessment

3. Cognitive expertise

4. Performance in practice (PIP)/practice quality improvement (PQI).

\section{American Board of Neurological Surgery}

Historically, the ABNS granted lifetime certificates. Starting in 1999, 10 year, time limited certificates were granted to new diplomates. The MOC program was initiated in January 2006. Participation is required of all diplomates holding time limited certificates.

Each 10 year MOC cycle consists of three 3 year mini cycles, plus a 10th year. All requirements must be met in each mini cycle. The cognitive examination may be taken in March during the third (final) mini cycle or during year 10. Because the MOC program was not implemented until 2006 and was phased in over 3 years, diplomates who were issued time limited certificates from 1999 to 2004 have truncated requirements that vary based on the year of primary certification.

Those with time limited certificates who do not successfully complete all elements of the MOC program during their 10 year cycle will have their certificates expire at the end of that cycle. The only mechanism to obtain a new certificate and be reinstated to diplomate status is to complete the requirements for initial certification again as they were previously described: both the primary and oral examinations.

To participate in neurosurgery MOC, one must have an ABNS certificate and a currently valid unrestricted license to practice medicine. Candidates may petition the board for exemptions or an extension of time in which to complete particular MOC requirements. These will be granted only in rare cases. The non-time limited certificates will not expire, regardless of participation in MOC. Holders of non-time limited certificates are not required to participate in MOC but are encouraged to do so.

In order to demonstrate evidence of professional standing, candidates must have a full unrestricted license to practice medicine, independent verification of unencumbered hospital privileges which is verified in every 3 year mini cycle, and a questionnaire completed by the chief medical officer or chief of surgery at the diplomate's primary hospital, also verified in every 3 year mini cycle.

In order to demonstrate evidence of commitment to lifelong learning and periodic self-assessment, one must earn at least 150 continuing medical education (CME) credits in each mini cycle with a minimum of 60 credits being category I neurosurgery. The diplomates and the ABNS are aided in this endeavor by the tracking of verified category I credits by the American Association of Neurological Surgeons. Additionally, in each mini cycle, the diplomate must participate in the self-assessment in neurological surgery (SANS) examination. Currently, there are three SANS examinations that will fulfill this requirement: regular SANS, spine, and pediatric. There is no specific SANS examination in cerebrovascular or endovascular neurosurgery at this time. The SANS is a web based tool and is managed by the Congress of Neurological Surgeons and available to MOC participants with a value of $24 \mathrm{CME}$ credits. The ABNS also recommends a fourth SANS, which covers compliance and similar pertinent issues. A safety module must be completed during each mini cycle.

Cognitive expertise is confirmed by passing a proctored examination once during each 10 year MOC cycle. In order to be eligible for the examination, the individual must be actively involved in MOC or have been successfully reinstated in the process if there was a period of non-participation. The examination may be taken in the last 4 years of the 10 year cycle. Individuals who do not pass may retake the examination in the next year, as long as they are still within their 10 year MOC cycle.

Evaluation of PIP is determined by submission of key cases and a communication assessment tool. In each mini cycle, participants select a key case from the available list and complete a web based module on 10 consecutive patients from their practice with similar neurosurgical problems. As case logs are being filled out, pop-ups give references to the literature about the case. At the end, graphs give feedback to the diplomate on his/ her outcomes, as well as the aggregate outcome of everyone who used that same key case. One of the key case modules is endovascular embolization of an anterior circulation aneurysm. A communication assessment tool using surveys of patients is still being developed. The goal is to assess communication skills during each mini cycle and generate a report for the participating neurosurgeon. ${ }^{8}$

\section{American Board of Radiology}

In order to demonstrate evidence of professional standing, diplomates are required to maintain active, valid, unrestricted medical licenses to practice in all of their own personal relevant 
locations. Current licenses will be checked both at the time of the computer based examination as well as through random checks. ${ }^{9}$ Diagnostic radiology certification must be maintained in order to also maintain neuroradiology subspecialty certification.

The ABR has implemented an integrated process linking ongoing certification with the requirements of MOC. This new process is called 'continuous certification'. The ABR certificates will no longer list an expiration date like a passport or driver's license. Certificates in diagnostic radiology, radiation oncology, or medical physics will now show a date of initial certification with a statement that ongoing validity of the certificate is contingent on meeting the requirements of MOC. This policy was implemented in 2012. The first full annual check to verify that diplomates are meeting all of their requirements will occur in March 2016. In addition to the materials available online, which are in a state of transition, this section was prepared in direct consultation with representatives of the ABR and utilizing an up to date brochure. ${ }^{10}$

Meeting the requirements of MOC will be reported on the ABMS public website (http://www.certificationmatters.org) and on the ABR website starting in 2013. This website will show whether or not each ABR diplomate is meeting the continuous certification requirements of MOC for each certificate held by the diplomate. Lifetime certified diplomates not participating in MOC will be reported as 'not required to participate in MOC'.

In order to demonstrate evidence of commitment to lifelong learning and periodic self-assessment, the diplomate must obtain a minimum of 75 CME credit hours, approved by the Accreditation Council for Continuing Medical Education (ACCME), over any 3 year period. Note that all of these CME credits must be in category I. Of the $75 \mathrm{~h}$, a minimum of $70 \%$ must be in specialty specific or in related areas. The remaining $30 \%$ can include additional specialty specific material as well as other relevant topics that, for example, might include risk management or ethics. Of the 75 category I CME credits in each 3 year period, one must earn 25 self-assessment CME credits to meet the requirement of periodic self-assessment. Self-assessment credits, available in a number of educational venues (eg, refresher courses, workshops, reading assignments, online offerings, etc), are usually offered by professional societies. All self-assessment credits are also qualified as category I CME and can also serve to fulfill that requirement. ${ }^{11}$

In order to demonstrate evidence of cognitive expertise at each annual look-back, ABR diplomates must have passed either the MOC practice profiled examination or an ABR initial certification examination (primary or subspecialty) within the past 10 years. The MOC examination may be taken multiple times, if necessary. The practice profiled examination recognizes that most radiologists are to some degree subspecialized. This is certainly true of neurointerventional radiologists. The ABR's practice profiled MOC examination was designed to be modular, allowing each radiologist to select a portion of his/her own examination content.

The ABR MOC examination contains two broad aspects: noninterpretive and interpretive skills. Non-interpretive skills content is designated by the ABR and includes such topics as patient safety, life support, professionalism/ethics, and principles of image quality. Study guides and an ABR produced noninterpretive skills syllabus are available on the ABR website. Areas of clinical content are selected by the diplomate and are based on the individual practice profile, which is completed during the examination registration process. Diplomates declare their practice profile through their online personal database, allowing each individual to specify the clinical areas in which he/ she wishes to be examined. Neurointerventionalists might choose to have all four areas of examination focus on neuroradiology. There are no specific modules for the neurointerventional area. Four hours in total are allotted for the examination. ${ }^{12}$

MOC part IV: evidence of evaluation and improvement of PIP (ABR's PQI). The ABR supports the 'plan-do-study-act' (PDSA) approach. This stepwise approach allows a diplomate to:

Plan: Identify an area within one's practice that could benefit from quality improvement and devise a relevant measure. As part of the planning process, one indicates how he or she would implement the measure and obtain the required data. As a last step in 'planning', the diplomate needs to set a goal for how the measure will be reached.

Do: Move forward with the plan and collect data.

Study: Assess how you have done in comparing your measure with the established goal. If your goal is not achieved, perform a root cause analysis of why not.

Act: Consider what can be done to address the root causes that are resultant from the study and develop an improvement plan to implement during the next PDSA cycle.

MOC participants are required to complete one PQI project every 3 years and attest to completion on their personal databases. Neuroradiologists should select projects that are relevant to their practice, are achievable in a practice setting, produce measurable results that are suitable for repeated measurements, and are able to effect quality improvement. The Society of NeuroInterventional Surgery might play a role in helping to develop products and model PQI projects. ${ }^{13}$

\section{American Board of Psychiatry and Neurology}

According to the website, the mission of the ABPN's MOC program is to advance the clinical practice of psychiatry and neurology by promoting the highest evidence based guidelines and standards to ensure excellence in all areas of care and practice improvement. The MOC program requires diplomates to participate in sanctioned self-assessment performance measures, identify perceived weaknesses in their knowledge, pursue learning activities tailored to areas that need to be strengthened, and develop quality improvement programs based on their clinical practice. The goal is for diplomates to reflect on their personal knowledge and performance, then commit to a process of improvement and re-evaluation of performance measures over a specified time frame that will ultimately lead to improved care for their patients.

Diplomates are responsible for their own self-assessment activities, continuing education credits, and practice improvement plans, and they can choose the learning tools that will best address their perceived needs, expand their expertise, and enhance the effectiveness and efficiency of their practice. Diplomates with certificates in the subspecialty of vascular neurology must also maintain certification in neurology in order to apply for recertification in the area of subspecialization. If certification in neurology lapses, one's certification in vascular neurology is no longer valid. ${ }^{14}$

Since October 1, 1994, all individuals achieving board certification by the ABPN are issued 10 year, time limited certificates. Certificates in vascular neurology, including those issued prior to October 1, 1994, are also 10 year, time limited certificates.

In order to demonstrate evidence of professional standing, the ABPN requires that diplomates must hold an active and unrestricted license to practice medicine. Such a license must be maintained even if the physician is out of the country for extended periods of time. All medical licenses must be unrestricted. 
In order to demonstrate evidence of commitment to lifelong learning and periodic self-assessment, diplomates of the ABPN are required to complete an average of 30 category I CME credits per year over the 10 year MOC cycle, either from organizations accredited by the ACGME or directly from the American Medical Association. Each self-assessment activity must cover new knowledge and/or current best practices in one or more of the competency areas, and provide feedback to the diplomate that can be used as the basis for focused CME, lifelong learning, and/or career development. That feedback must include the correct answer and recommended literature resources for each question, and (by 2014) comparative performance to peers. This requirement began in 2010 for those applying for 2011 MOC examinations. Beginning in 2014, diplomates are required to use only ABPN approved products for self-assessment activities.

When this component is fully implemented in 2014, an average of eight of the CME credits per year (averaged over 2-5 years) should involve self-assessment with proscribed times during the 10 year cycle when the first and second self-assessment activity occurs. Feedback must include comparative performance to peers. Self-assessment examinations include a minimum of 25 questions, and $2 \mathrm{CME}$ credits are awarded for each activity.

The self-assessment examination occurs before the activity (http://www.abpn.com/psych_moc.html).

Diplomates of the ABPN are required to complete an average of 30 specialty or subspecialty category I CME credits accredited by the ACCME per year over the 10 year MOC cycle. The CME must be relevant to the specialty in which the diplomate is certified which would, for example, be relevant to subspecialty certified vascular neurologists. The same CME credits can be used to satisfy the MOC requirements for multiple specialties and subspecialties. This requirement was phased in beginning in 2006 for those applying for 2007 MOC examinations.

When this component is fully implemented in 2014, 150 CME credits must be earned during each 5 year block of the 10 year MOC cycle for a total of 300 category I CME credits over the 10 year MOC cycle.

In order to demonstrate evidence of cognitive expertise, ABPN diplomates must pass an examination prior to the expiration date on their certificates. To sit for the examination, all MOC requirements must be satisfied at the time the application is submitted. A passing score on the cognitive examination extends the renewal date of the certificate 10 years out from the cognitive examination. The examination is computer based, multiple choice, and is meant to be relevant to practice and clinically oriented. According to the ABPN website, to prepare for the MOC cognitive examinations, a diplomate "should keep current with research and developments in their respective field, read specialty specific journals and practice guidelines, and attend relevant CME programs".

In order to demonstrate evidence of the evaluation of PIP, the ABPN has a unique two part quality improvement program designed for 'clinically active' physicians. These include chart review and second party external review. Diplomates will be required to complete three PIP units, each consisting of both a clinical module (chart review) and a feedback module (second party external review).

If a diplomate participates in an institutional quality improvement program that involves chart review, this may fulfill the clinical module. If a diplomate participates in peer review in his/ her clinical setting, that institutional activity may also fulfill the PIP feedback module criteria. This requirement is currently slated to begin in 2013 for those applying for 2014 MOC examinations. Beginning in 2014, diplomates are required to use only ABPN approved products for PIP activities. ${ }^{15}$

\section{SUMMARY AND CONCLUSION}

The ABNS, ABR, and ABPN have all embraced MOC as a core part of their ethos and practice. The vast majority of Society of NeuroInterventional Surgery members are certified by one of these three organizations. Depending on the year of primary certification, the parent board, and decisions regarding subspecialization such as the neuroradiology CAQs, many members are likely already participating in MOC programs. While some may argue with the desirability of the four core competencies of MOC generally, MOC nonetheless represents a new challenge for non-time limited certificate holders of the parent organizations. The future likely includes further expansion of MOC programs as hospitals, state licensure boards, and other organizations make participations in MOC a requirement of practice.

Acknowledgements We would like to acknowledge the administrators and volunteer physicians of the $A B N S, A B R$, and $A B P N$ who contributed their time, knowledge, and perspective to the completion of this document on behalf of their respective societies. Specifically, Mary Louise Spencer, Executive Director of ABNS, Dr Jennifer L Bosma and Donna Breckenridge of $A B R$, and Dr Larry R Faulkner, President and CEO of ABPN.

Contributors All authors contributed to this manuscript.

Competing interests VCT is a Director of ABNS. GJB is the Executive Director of ABR.

Provenance and peer review Commissioned; not externally peer reviewed.

\section{REFERENCES}

1 ABNS. About the American Board of Neurological Surgeons-ABMS. Woodbridge, CT, 2012. http://www.abns.org/content/about_abns.asp (accessed 12 Oct 2012).

2 ABNS. Certification Process. Woodbridge, CT, 2012. http://www.abns.org/content/ primary_certification_process.asp (accessed 12 Oct 2012).

3 ABR. About the American Board of Radiology. Tucson, AZ, 2012. http://www. theabr.org/about-landing (accessed 12 Oct 2012).

4 ABR. Core and Certifying Examinations. Tucson, AZ, 2012. http://www.theabr.org/ eof-overview (accessed 12 Oct 2012).

5 Hirsch JA, Meyers PM. Maintenance of certification: historical context. J Neurointerven Surg 2013;5:612-14.

6 ABPN. What is the ABPN? Bufallo Grove, IL, 2012. http://www.abpn.com/ what_is_abpn.html (accessed 12 Oct 2012).

7 ABPN. Certification in Vascular Neurology. Bufallo Grove, IL, 2012. http://www. abpn.com/cert_vn.html (accessed 12 Oct 2012).

8 ABNS. Maintenance of Certification. Woodbridge, CT, 2012. http://www.abns.org/ content/moc_info.asp (accessed 12 Oct 2012).

9 ABR. Maintenance of Certification in Neuroradiology. Tucson, AZ, 2012. http:/l www.theabr.org/moc-neuro-comp1 (accessed 12 Oct 2012).

10 ABR. Maintenance of Certification (MOC). What it is and what it means for you. Chicago, IL: American Board of Radiology, 2012.

11 ABR. Self Assessment Modules in Maintenance of Certification. Tucson, AZ, 2012. http://www.theabr.org/moc-neuro-comp2 (accessed 12 Oct 2012).

12 ABR. Examination in Maintenance of Certification. Tucson, AZ, 2012. http://www. theabr.org/moc-neuro-comp3 (accessed 12 Oct 2012).

13 ABR. Practice Quality Improvement (PQI). Tucson, AZ, 2012. http://www.theabr.org/ moc-neuro-comp4 (accessed 12 Oct 2012).

14 ABPN. Maintenance of Certification. Bufallo Grove, IL, 2012. http://www.abpn.com/ moc.html (accessed 12 Oct 2012)

15 ABPN. Performance in Practice. Bufallo Grove, IL, 2012. http://www.abpn.com/ moc_10yrmoc.html (accessed 12 Oct 2012). 